\title{
Enhancing the alternative cellular energy (ace) pathway in the prevention of zika virus induced illness
}

\begin{abstract}
The Public Health response to Zika virus induced microcephaly is largely directed at developing a protective vaccine and in devising methods for restricting human exposure to infected mosquitoes. These efforts do not address the urgent need to treat currently infected, or soon to be infected pregnant women. A key to such a strategy is understanding the alternative cellular energy (ACE) pathway. This pathway was initially identified in the defense against stealth adapted viruses. These viruses are derivatives of conventional viruses with deletion or mutation of the genes coding the major antigens normally targeted by the cellular immune system. It was subsequently shown that the ACE pathway is also effective in suppressing other viruses, including herpes simplex virus (HSV) and the human immunodeficiency virus (HIV). This review is intended to briefly explain the ACE pathway and to suggest a relatively easy initial approach at potentially enhancing the ACE pathway. It is the regular consumption of water with greatly increased dynamic (kinetic) activity induced by a natural environmental energy termed KELEA (kinetic energy limiting electrostatic attraction).
\end{abstract}

Keywords: Zika virus, Alternative cellular energy, ACE, KELEA, Water, Anti-vira immunity, Micocephaly, Inflammation
Volume 3 Issue 4 - 2016

\author{
W John Martin \\ Institute of Progressive Medicine, USA
}

Correspondence: W John Martin, Institute of Progressive Medicine, 1634 Spruce Street, South Pasadena CA 91030, USA, Tel 626-616-2868 Email wjohnmartin@ccid.org

Received: June 23, 2016 | Published: June 23, 2016
Abbreviations:KELEA, Kinetic Energy Limiting Electrostatic Attraction; ACE, Alternative Cellular Energy; ICE, Insufficiency of Cellular Energy; CTL, Cytotoxic T Lymphocyte; HCMV, Human Cytomegalovirus; SCMV, African Green Monkey Simian Cytomegalovirus; HSV, Herpes Simplex Virus; CPE, Cytopathic Effect; UV, Ultraviolet

\section{Introduction}

The concept of stealth adaptation of viruses has yet to be generally accepted among either virologists or immunologists. ${ }^{1}$ Virologists generally doubt that a virus can survive if several of its major genes are deleted or mutated. Most immunologists do not fully appreciate that as a corollary of the Clonal Selection Theory of Immunology. ${ }^{2}$ virus infected cells need to present multiple copies of only a limited diversity of virus components for effective immune recognition by cytotoxic T lymphocytes (CTL). A striking example of restricted antigen expression occurs with human cytomegalovirus (HCMV). Over half of the responding CTL target a single antigen coded by the UL83 gene, with the majority of the remaining CTL targeting either of two additional antigens. ${ }^{3}$ Deletion or mutation of the three genes, along with other adaptations to retain virus replication can result in a cell damaging (cytopathic) virus, which does not evoke a cellular immune response. ${ }^{1}$ Stealth adaptation is a generic immune evasion mechanism potentially occurring with all types of pathogenic viruses.

The initially described stealth adapted cytomegalovirus was not of human origin, but rather derived from African green monkey simian cytomegalovirus (SCMV). ${ }^{4}$ This finding was eschewed by Public Health officials since it implied human infection as being a consequence of the use of SCMV infected monkeys in the production of live polio virus vaccines. It also raised the issue of whether rhesus cytomegalovirus contamination of the experimental polio vaccine tested in African chimpanzees led to the transformation of chimpanzee immunodeficiency virus (SIV) to human immunodeficiency virus (HIV). ${ }^{5}$
Stealth adapted SCMV induces a severe, acute illness in cats, from which there is recovery in spite of the lack of accompanying inflammation. ${ }^{6}$ Similarly, in vitro cultures of stealth adapted viruses will commonly show cellular repair, especially if not re-fed with fresh tissue culture medium. ${ }^{7}$ Both sets of observations point to a non-immunological cellular repair process. The tissue culture suppression of the virus cytopathic effect (CPE) is mediated by an induced change in the tissue culture medium. This occurs with the cellular production of particulate materials with energy transducing activities. When released from infected cells, the materials can selfassemble into larger particles, which are commonly pigmented; as well as forming into fibers and other complex structures. Both intracellular and extracellular materials are fluorescent, especially when stained with certain dyes, including neutral red. The particles are electrostatic, occasionally ferromagnetic and can induce slowly evolving vapor bubbles in water. ${ }^{7}$ Similar complex structures can be seen in brain biopsies of stealth virus infected patients. ${ }^{8}$ A notable feature of the cells producing these materials is the marked disruption of the cell's mitochondria, the major source of cellular energy from food metabolism. It was proposed that these cell derived materials are providing a source of cellular energy distinct from that of food metabolism. They were, accordingly termed alternative cellular energy (ACE) pigments and the non-mitochondria source of energy referred to as the ACE pathway. ${ }^{7}$

The addition of ACE pigments to the tissue culture medium used for refeeding stealth adapted virus infected cultures prevented the reactivation of the CPE normally seen upon refeeding of the cultures with fresh medium. ${ }^{7}$ The reactivation process could also be suppressed using a nominally homeopathic product called Enercel (unpublished data). When administered by injection, Enercel and a similar product termed HANSI (homeopathic activator of the system immune) have produced clinical benefits in a number of illnesses, including cancer.9 AIDS. ${ }^{10}$ tuberculosis. ${ }^{10}$ and childhood diarrhea. ${ }^{11}$ 
These studies relate to a fluid activation process, which was demonstrated with water and subsequently shown with other fluids. ${ }^{12-18} \mathrm{An}$ informative assay with water is to observe the dissolving pattern of fine particles of neutral red dye sprinkled onto the water. With water with minimal or no activation, the particles essentially remain stationary on the surface of the water, with a slowly expanding circular area of dissolved dye forming about each of the particles. This static pattern contrasts markedly with the pattern seen when neutral red dye particles are sprinkled onto highly activated water. The dye particles pass through the surface of the water due to lower surface tension. They also readily move in a linear to-and-fro manner forming streaks of dissolved dye. Residual non-dissolved dye particles will commonly form into small, loose clusters. Individual particles are periodically rapidly repelled from the cluster, only to slowly return back towards the cluster, to be repelled yet again. Neutral red dye sprinkled onto regular ethanol provides a suitable positive control for the actions of activated water on neutral red including fluorescence of the dye. Another assay to distinguish activated from regular water is the increased rate of weight loss in closed but not completely sealed containers. Up to 50 fold differences in weight loss can be observed over several hours using this relatively simple, quantitative assay of volatility.

It became apparent using these and other assays that the kinetic activity of water was being quantitatively increased. Activation of water can be easily achieved using either of two basic approaches. ${ }^{18}$ The first involves the addition of various water activating products. The second is simply placing the water within certain energy fields. The water activating products are essentially dipolar compounds with separated electrical charges. They appear to attract and then release an environmental water activating energy, possibly in an oscillating manner. As listed elsewhere. ${ }^{18}$ the activating compounds can be either water soluble or insoluble. With soluble compounds, once the water is activated, the activating compound can be removed by zero residue filtration or by repeated dilutions, as in homeopathy. Moreover, if water is sufficiently activated, the now separated charges can seemingly directly attract further energy from the environment. This can lead to sustained activation and can also induce the activation of added water, as in the dilutions used in homeopathy.

The underlying principle of energy field activation of water is the repetitive on-off switching of various electrical devices. Several of the devices are similar to those utilized within the field of complementary and alternative medicine. ${ }^{19}$ These devices appear to attract and then release the environmental energy in a manner that can lead to activation of nearby water. For example, one method is to focus opposing, fluctuating light sources towards a central area. ${ }^{20}$ Water placed within the area of opposing lights will become activated over time. Water activating dipolar compounds as well as activated water by itself can also slowly transfer energy to nearby water, without the need for direct contact.

The electrical charge attracting energy was proposed to comprise a fundamental force of Nature to prevent electrostatically attracted opposite electrical charges from fusion and possible annihilation. It may also explain the repulsion observed between like electrical charges. The term KELEA (kinetic energy limiting electrostatic attraction) was chosen to describe this energy. It is likely to be identical to the radiant or impulse energy proposed by Nikola Tesla. ${ }^{21}$ The term KELEA is somewhat more informative and is consistent with unpublished studies on altered electrostatic attraction. The effect of KELEA absorbed into water and other fluids is basically explainable as a weakening of the electrostatic, hydrogen bonding between molecules, together with added kinetic activity of the less bonded molecules. ${ }^{22}$

The ACE pathway is, therefore, regarded as energy added to the body's fluids from the absorption of KELEA. It facilitates many of the body's functional activities and is probably more than simply an additive to the cellular energy provided by the metabolism of food. Indeed, simple calculations confirm that food metabolism is inadequate to explain the daily energy expenditure by humans. More interesting is the likelihood that aspects of brain activity may be uniquely dependent upon the ACE pathway, rather than on the energy derived from food. The electrical activity of the brain may even directly contribute to the ACE pathway. ${ }^{23}$ in a positive feedback manner.

The ACE pathway can potentially help restore health in illnesses attributed to an insufficiency of cellular energy (ICE) from food metabolism. ${ }^{18}$ This can arise in the following circumstances:

i. Inadequate delivery of oxygen to the tissues as in chronic obstructive pulmonary disease (COPD);

\section{ii. Impaired blood supply as in cardiovascular disease;}

iii. Cellular biochemical cellular defects, as in diabetes and probably in many cancers that fail to undergo apoptosis; and

iv. Increased energy demands as in infections and as required for tissue regeneration following injuries.

Experimental support for enhancing the ACE pathway in the treatment of infectious diseases has already been obtained. Enercel is effective in suppressing tropical diarrhea in children, including cases due to rotavirus. ${ }^{11}$ It suppresses tuberculosis and HIV infections in AIDS patients. ${ }^{10}$ Skin lesions caused by herpes simplex virus (HSV), herpes zoster virus (HZV) and human papillomavirus (HPV) will show striking ultraviolet (UV) light induce fluorescence after being stained with neutral red dye. Fluorescence will commonly also begin to occur upon UV illumination of ACE pigment containing lesions that are not directly treated with the neutral red dye. All of the lesions undergo expedited healing, presumably through an overall activation of the ACE pathway. ${ }^{24} \mathrm{UV}$ activation of neutral red dye in activated water was also shown to be beneficial in the suppression of autism, a stealth adapted virus induced illness. ${ }^{25}$ Rather than pursuing these earlier protocols, preliminary data now suggest a likely benefit from simply consuming KELEA activated drinking water. For pregnant women, it is initially preferable to use water activated by exposure to an energy field, rather than water with added chemical components. It is also reasonable, with Institutional Review Board (IRB) approval, to use water with minimal or no detectable residual chemicals, as in the production of certain effective homeopathic formulations. Controlled clinical studies using the KELEA activated water in medical conditions other than Zika infections will also be helpful to confirm efficacy and to optimize attainable benefits.

The issue as to why Zika virus is now associated with microcephaly remains controversial. Possibly earlier cases of microcephaly were simply missed or the Zika virus has undergone critical mutations. Data do not support either contention. Another suggestion is that coinfection of the placenta with stealth adapted viruses is allowing for the transplacental passage of the Zika virus to the developing fetus. ${ }^{26}$ If this mechanism is operative, it would further justify therapeutic approaches aimed at enhancing the ACE pathway.

In conclusion, an understanding of the ACE pathway provides a rational approach for preventing Zika virus induced illnesses. 
Consuming KELEA activated drinking water is the most practical of the various means currently available to enhance the ACE pathway. It is particularly suitable for testing in pregnant women who are either currently Zika virus infected or at risk of becoming infected. Public Health authorities could help with the initial testing and with the wider implementation of this approach to help prevent the brain damage otherwise expected to occur in the infants born to many of these mothers. Successful clinical trials in Zika virus infected patients will help encourage wider clinical applications of ACE pathway based therapies. Such efforts should include the prevention of stealth adapted virus illnesses, including autism.

\section{Acknowledgements}

The Institute of Progressive Medicine is a component of MI Hope Inc., a non-profit public charity.

\section{Conflicts of interest}

None.

\section{References}

1. Martin WJ Stealth adaptation of viruses: Review and updated molecular analysis on a stealth adapted African green monkey simian cytomegalovirus (SCMV). Journal of Human Virology \& Retrovirology 2014;1(4):00020.

2. Burnet FM The Clonal Selection Theory of Acquired Immunity. Nashville: Vanderbilt University Press, USA, pp. 1959;236.

3. Wills MR, Carmichael AJ, Mynard K, Jin X et al. The human cytotoxic $\mathrm{T}$-lymphocyte (CTL) response to cytomegalovirus is dominated by structural protein pp65: frequency, specificity, and $\mathrm{T}-$ cell receptor usage of pp65-specific CTL. J Virol. 1996;70(11):7569-7579.

4. Martin WJ, Ahmed KN, Zeng LC et al. African green monkey origin of the atypical cytopathic stealth virus isolated from a patient with chronic fatigue syndrome. Clin Diagn Virol. 1995;4(1):93-103.

5. Martin WJ Chimpanzees inoculated with cytomegalovirus contaminated polio vaccines may explain origin of HIV-1. Journal of Human Virology \& Retrovirology. 2015;2(2):00035.

6. Martin WJ, Glass RT Acute encephalopathy induced in cats with a stealth virus isolated from a patient with chronic fatigue syndrome. Pathobiology. 1995;63(3):115-118.

7. Martin WJ Stealth virus culture pigments: A potential source of cellular energy. Exp Mol Path. 2003;74(3):210-223.

8. Martin WJ Complex intracellular inclusions in the brain of a child with a stealth virus encephalopathy. Exp Mol Path. 2003;74(3):179-209.

9. Martin WJ, Laurent D Homeopathy as a misnomer for activation of the alternative cellular energy pathway: Evidence for the therapeutic benefits of Enercel in a diverse range of clinical illnesses. International Journal Complementary \& Alternative Medicine. 2015;2(1):00045.

10. Dubrov V, Dubrova T, Christner D et al. Alternative cellular energy based therapy using EnercelÔ in advanced AIDS patients co-infected with tuberculosis and treated in Chernigov, Ukraine. Journal Human Virology \& Retrovirology. 2015;2(6):00061.
11. Martin WJ Alternative cellular energy (ACE) based therapy of childhood diarrhea. In Stealth Adapted Viruses; Alternative Cellular Energy (ACE) \& KELEA Activated Water. Author House, IN, pp. 2014;112-131.

12. Martin WJ KELEA activated water leading to improved quantity \& quality of agricultural crops. Advances in Plants \& Agriculture Research. 2014;2(1):00033.

13. Martin WJ KELEA activation of water and other fluids for health, agriculture and industry. Journal of Water Resource and Protection. 2015;7(16):1331-1344.

14. Martin WJ Alternative cellular energy pathway therapy using KELEA activated water. International Journal Complementary \& Alternative Medicine. 2015;2(2):00051.

15. Martin WJ Therapeutic Potential of KELEA activated water. International Journal of Complementary \& Alternative Medicine. 2015;1(1):00001.

16. Martin WJ Alternative cellular energy as a unifying concept in complementary alternative medicine. International Journal Complementary \& Alternative Medicine. 2015;1(4):00022.

17. Martin WJ Deconstructing medicine. The alternative cellular energy pathway. British Journal of Medicine \& Medical Research. 2016;11(8):1-6.

18. Martin WJ Preparing and using KELEA activated water to enhance the alternative cellular energy (ACE) pathway in the therapy of multiple illnesses. International Journal Complementary \& Alternative Medicine. 2016;3(1):00059.

19. Martin WJ Cancer as an insufficiency of cellular energy (ICE): Therapeutic approaches based on enhancing the alternative cellular energy (ACE) pathway. International Journal Complementary \& Alternative Medicine. 2016;3(3):00074.

20. Martin WJ Interacting light paths attract KELEA (kinetic energy limiting electrostatic attraction) and can lead to the activation of water. Open Journal of Biophysics. 2015;5(4):115-121.

21. Tesla N Tesla Patent 685,958 Method of Utilizing Radiant Energy. United States Patent, USA. 1901

22. Martin WJ KELEA: A natural energy that seemingly reduces intermolecular hydrogen bonding in water and other liquids. Open Journal of Biophysics. 2015;5(3):69-79.

23. Martin WJ Is the Brain an Activator of the Alternative Cellular Energy (ACE) Pathway? International J Complementary \& Alternative Medicine 2015;1(1):00002.

24. Martin WJ, Stoneburner J Alternative cellular energy (ACE) pathway activation as the mode of action of neutral red dye phototherapy of human viruses. J Human Virology \& Retrovirology. 2014;1(4):00019.

25. Martin WJ Alternative cellular energy (ACE) activation as natural therapy for autism. In Stealth Adapted Viruses; Alternative Cellular Energy (ACE) \& KELEA Activated Water. Author House, IN, pp 2014;89-111.

26. Martin WJ Co-infection with stealth adapted viruses may explain Zika virus associated microcephaly. Journal Virology \& Retrovirology. 2016;3(1):00080. 